\title{
La mobilité des ménages périurbains au risque des crises économiques et environnementales
}

Florent Le Néchet*, UMR T 9403 LVMT - UPEM Maître de conférences; Hélène Nessi, UMR LAVUE Mosaïque, MCF à Nanterre Paris Ouest-la Défense ; Anne Aguiléra, UMR T 9403 LVMT - IFSTTAR chercheuse

* Auteur correspondant: Le Néchet, F., Université Paris-Est, Laboratoire Ville Mobilité Transport, UPEMLV : 5 boulevard Copernic, Cité Descartes F 77454 Marne-la-Vallée cedex 2 France, florent.lenechet@u-pem.fr

\section{Résumé}

À partir de deux enquêtes, cet article explore les marges de manœuvre dont disposent les ménages périurbains franciliens pour faire évoluer leurs pratiques de mobilité, et les conditions à mettre en œuvre pour favoriser ces changements. Quatre évolutions favorables à une réduction de l'usage de la voiture se dégagent : (1) celle d'une réorganisation profonde du système de transports en commun, (2) celle d'une flexibilisation des liens entre travail et lieu de travail via l'option du télétravail à domicile, (3) celle d'une hausse sensible du prix des carburants et (4) celle du développement des ressources locales, notamment à l'échelle communale, permettant la poursuite de l'inscription de la vie quotidienne des périurbains dans leur territoire de résidence.

Mots-clés : Périurbain, automobile, mobilité durable, crise économique, île de France

\begin{abstract}
:
Based on two surveys, this article explores the potential for changing the travel behaviour of inhabitants in periurban areas in the Paris Region, and the conditions that could foster behavioural changes towards more sustainable practices. Four trends appear favourable to changes in travel behaviour: (1) a profound reorganization of public transport, (2) more flexible working conditions (home-based teleworking), (3) a significant increase in oil price and (4) the development of local resources, primarily at the municipality scale, which should foster the increasing inscription of everyday activities in the municipality of residence.
\end{abstract}

Key-words: periurban areas, automobile, sustainable mobility, economic crisis, Paris Region 


\section{Introduction}

Organiser la mobilité durable dans le périurbain ${ }^{1}$, situé à l'interface entre les ressources urbaines et rurales, constitue un enjeu fort de politique publique. Selon les chiffres du Recensement de 2008, 19\% des Français habitent dans une commune périurbaine, c'est-àdire située dans la couronne d'une aire urbaine selon la définition établie par l'INSEE (Brutel et Levy, 2011). Entre 1999 et 2008, la périurbanisation s'est par ailleurs poursuivie autour de la majorité des aires urbaines, en particulier les plus grandes (Baccaïni et Sémécurbe, 2009 ; Floch et Levy, 2011).

D'importantes évolutions en matière démographique, économique et de modes de vie ont marqué les territoires périurbains. Leur structure démographique s'est ainsi largement diversifiée, avec un accroissement des célibataires et des familles monoparentales (Aragau et al., 2011). Les commerces et services aux populations se sont par ailleurs développés, comme le montrent notamment les travaux de Beaucire et Chalonge (2007). Des changements de modes de vie ont accompagné ces dynamiques géographiques (Berger et al. 2014), qui donnent la possibilité aux ménages périurbains de valoriser simultanément l'accessibilité à un certain nombre d'aménités métropolitaines de niveau élevé (centre d'emploi, commerce de proximité, commerce spécialisé, santé) et l'accès à des ressources de proximité, commerces, services, mais aussi ressources naturelles (comme les massifs forestiers).

Si les ménages périurbains continuent de faire des déplacements plus longs (en distance) et principalement en automobile, on observe en Île-de-France une stabilisation des distances et de l'usage de la voiture, de sorte que les différences de mobilité avec les autres Franciliens s'estompent progressivement. En particulier, les périurbains tendent à relocaliser leurs activités dans des périmètres plus restreints (Bouleau et Mettetal, 2013 et 2014), à l'exception des activités professionnelles du fait de la persistance de la concentration des emplois dans les pôles urbains (Floch et Levy, 2011).

Malgré ces évolutions, le mode de vie périurbain reste largement construit autour de l'usage de la voiture individuelle, l'accessibilité aux transports en commun y étant de fait médiocre : en région lle-de-France, hors Paris intra-muros, moins de $20 \%$ de la population habite à moins de 1 kilomètre à vol d'oiseau d'une station du réseau ferré (Le Néchet, 2012). L'autosolisme des ménages périurbains, défini comme usage individuel de l'automobile, demeure donc un enjeu majeur de politique publique, dont la dimension n'est pas seulement environnementale, mais également sociale et économique. Parmi les scénarios d'évolution des prix du pétrole de Donovan et al. (2008), l'un conduit en effet à un triplement du prix à horizon 2040 par rapport à 2007, ce qui aurait des conséquences particulièrement importantes en matière de précarité énergétique des périurbains (Saujot, 2012).

\footnotetext{
1 Le terme " périurbain » est employé tout au long de l'article dans le sens d'espaces à faible densité, situés en périphérie des villes, définition qui n'épouse pas stricto sensu la définition des couronnes périurbaines proposée par l'INSEE. Toutefois les chiffres donnés en introduction concernent le périmètre INSEE des couronnes périurbaines.
} 
De nombreuses recherches se sont intéressées au cours des dernières années aux conditions favorables à des changements des comportements de mobilité, en particulier dans les espaces à faible densité. Une première série de travaux identifie les facteurs socio-économiques et de forme urbaine (à une échelle locale ou plus large) qui expliquent les comportements de mobilité, et notamment de choix modal (Ewing et Cervero, 2010 ; Le Néchet et Aguiléra, 2012). Les données utilisées proviennent généralement des enquêtes sur la mobilité des ménages ou les recensements de population. Les résultats obtenus servent à formuler des préconisations en matière de politiques de transport et d'aménagement : densification, meilleur équilibrage habitat-emploi, ou encore développement des transports en commun.

Une deuxième série de recherches s'appuie sur l'évaluation de politiques d'aménagement ou de transport (par exemple des mesures de restriction de l'usage de la voiture, la mise en place du télétravail dans une entreprise, etc.). Les résultats montrent notamment que le développement et l'amélioration des offres de transport ne suffisent pas à obtenir des changements comportementaux significatifs dans les territoires périurbains, où la congestion automobile est faible et où les destinations sont très diffuses (Laugier, 2012). Par ailleurs, les mesures de restriction de l'usage de la voiture font émerger de fortes inégalités sociospatiales (Orfeuil, Ripoll, 2015).

Enfin une troisième série de travaux, dans laquelle s'inscrit cet article, s'intéresse aux modalités d'évolution des comportements individuels, à travers des approches souvent plus qualitatives et parfois basées sur des expérimentations (accompagnement au changement) (Bonsall, 2009 ; Huyghe, 2015). Il s'agit de comprendre les freins et les moteurs, économiques, organisationnels, psychologiques ou relevant des habitudes et des normes sociales (Bamberg et al., 2011 ; Kaufmann et al., 2010), aux changements (Schwanen et al., 2012). Il en ressort que les évolutions en matière de mobilité se heurtent à de nombreux freins, qui incluent la méconnaissance de l'existence ou du fonctionnement de toutes les alternatives possibles, la force des habitudes, une déformation de la représentation des autres modes de transports ou encore à un manque d'expérience dans la pratique d'autres modes (Rocci, 2009 ; Vincent, 2008). La montée des préoccupations environnementales ne semble par ailleurs pour l'instant pas suffisante pour faire changer les pratiques de mobilité (Barr et Prillwitz, 2011 ; Draetta, 2003 ; Lorenzoni et Langford, 2001 ; Rocci, 2009).

Cet article explore les potentiels de changement des pratiques de mobilité dans le périurbain francilien en étudiant les marges de manœuvre et les stratégies envisagées dès aujourd'hui par les résidents pour diminuer leurs usages de l'automobile. II ne s'agissait pas de placer les individus que nous avons enquêtés dans un objectif radical de sevrage automobile, qui semble peu réaliste au moins à moyen terme, mais plutôt de permettre de révéler les chemins vers des pratiques plus "sobres». Par pratiques plus sobres, nous entendons plusieurs types d'évolutions : des changements modaux vers des modes alternatifs à la voiture thermique, 
incluant le passage à un véhicule électrique ou hybride, une baisse des distances parcourues en voiture, et enfin une croissance des usages partagés de l'automobile.

Nous avons considéré trois catégories de facteurs. Il s'agissait ici d'évaluer dans quelle mesure les ménages périurbains les considéraient comme favorables à des pratiques de mobilité plus sobres, afin d'en tirer des enseignements en matière de politiques publiques locales. Ces facteurs, présentés en première partie de cet article, concernent :

- Les conditions de la mobilité, en termes de coûts des carburants, mais aussi d'offre locale de transport en commun, d'aménagements pour favoriser les mobilités douces (marche et vélo), de services de mobilité et enfin d'alternatives à la voiture thermique ;

- La proximité aux ressources nécessaires aux activités du quotidien (commerces et services) ;

- L'usage des $\mathrm{TIC}^{2}$. Notre réflexion, moins développée que sur les deux points précédents, a principalement porté sur le rôle que peut ou pourrait jouer le télétravail à domicile ainsi que le e-commerce sur les déplacements physiques et le recours à la voiture ;

La deuxième partie de l'article expose la méthodologie des enquêtes qui ont été réalisées auprès d'un peu plus de 300 habitants de plusieurs communautés de communes situées dans le périurbain francilien. La troisième partie rend compte des résultats et identifie les marges de manœuvre pour l'action publique dans ces territoires.

II convient d'être prudent, car si cet article s'inscrit dans une recherche qui décrit de manière approfondie les changements actuels à l'œuvre dans les pratiques de mobilité, les résultats qui y sont présentés ne montrent pas le changement, mais la manière dont les ménages se projettent dans des changements futurs. L'objectif est bien de déterminer si des stratégies sont d'ores et déjà envisagées par les ménages, quelles sont ces stratégies, et comment les pouvoirs publics pourraient favoriser des changements vers une mobilité moins dépendante de la voiture dans le périurbain.

\section{Identifier le potentiel de changement et d'adaptation des pratiques de mobilité des ménages}

\subsection{Les conditions de la mobilité}

Un premier facteur que nous avons voulu tester concerne la manière dont les ménages concilient un mode de vie très dépendant de la voiture avec des contraintes budgétaires susceptibles de se durcir. Les travaux de Hughes et al. (2008) montrent dans le contexte

\footnotetext{
2 dont les relations avec la mobilité demeurent largement indéterminées dans la littérature, trois registres étant envisagés : la substitution (les TIC réduisent les déplacements), la complémentarité et enfin l'induction (les TIC augmentent le besoin de mobilité) (Aguiléra et al., 2012).
} 
américain que l'élasticité de la demande de transport par rapport au prix du carburant a fortement baissé entre 1970 et 2000 (l'élasticité correspond à la variation du total de la distance parcourue en automobile lorsque le prix augmente). Deux principales hypothèses explicatives de cette tendance sont dégagées par les auteurs. La première est que l'augmentation générale des revenus des ménages rend à proportion le coût des déplacements plus faible, rendant mécaniquement moins nécessaire un changement de comportement dans un contexte d'augmentation des coûts de transport. L'autre hypothèse est celle d'une dépendance accrue à l'automobile pendant la période d'étude qui fait que les ménages sont moins capables de se tourner vers d'autres formes de déplacement (par exemple: suppression de services de transports collectifs). Le présent article explore les marges de manœuvre et stratégies des habitants par rapport à ce point, dans un contexte où une augmentation sensible ( $+50 \%$ pour le prix du gazole, augmentation un peu plus faible pour l'essence) a eu lieu entre janvier 2009 et janvier 2013, juste avant la réalisation de nos enquêtes (données du Conseil National Routier, 2014). Cet épisode de hausse a pu conduire certains ménages à modifier leurs pratiques de mobilité, ou en tous les cas à entamer une réflexion sur les stratégies possibles en cas de nouvelle hausse.

Le rôle des conditions de mobilité sur les pratiques des périurbains a également été étudié au regard d'une amélioration sensible de l'offre de transports en commun. L'équilibre offre/demande de transport conduit dans le périurbain à l'utilisation presque exclusive de l'automobile pour les déplacements non locaux, du fait notamment d'un maillage bien plus important du territoire par ce mode et conséquemment de temps de trajets plus courts.

La question des transports en commun doit être reconsidérée dans la mesure où le périurbain devient avec le temps à la fois plus autonome fonctionnellement, c'est-à-dire avec une demande de déplacement interne plus forte, et également davantage polarisé par des pôles secondaires (Berroir et al., 2007) vers lesquels il est plus facile d'organiser le rabattement en transport collectif. Cependant, cette solution présente un coût important et n'est pas forcément facile à mettre en œuvre ni souhaitable pour la collectivité, en particulier dans un contexte de tarissement des financements publics. Il est également possible d'agir sur l'offre à moindre coût en proposant une amélioration de la lisibilité de l'offre et de l'information voyageur ou en proposant une offre de type transport à la demande dans les territoires les moins denses (Castex, 2007). Une dernière option est d'agir sur la demande de transport en visant à renverser l'image de lenteur et d'inconfort des transports collectifs. Des dispositifs d'accompagnement sont expérimentés (Rocci, 2009) afin de permettre à des ménages qui ne connaîtraient pas l'offre existante de l'utiliser plus facilement. Il est également possible d'intervenir en amont sur l'image environnementale des transports collectifs, à travers du marketing ciblé et à plus long terme par une politique d'éducation (Banister, 2008).

Au regard de nos enquêtes confirmant la dispersion spatiale, notamment due une baisse des liens avec Paris pour les trajets domicile-travail (Conti, 2012), et une large demande de 
flexibilité dans les emplois du temps (pour les activités non contraintes), nous avons investigué deux pistes : une meilleure adaptation des transports en commun en termes premièrement de trajets, et deuxièmement d'horaires. L'influence potentielle de la mise à disposition de services de mobilité a également été testée : au moment de la réalisation des enquêtes, il existait principalement des services de transport à la demande (toutefois réservées aux personnes âgées ou handicapées), et le terme de co-voiturage commençait à passer dans le langage courant. Nous avons donc centré notre réflexion sur ces deux catégories de services.

Nous avons, par ailleurs, cherché à évaluer si des améliorations en matière d'itinéraires pour les vélos et les piétons pouvaient aider les ménages à adopter des mobilités plus sobres, non seulement en induisant des transferts modaux mais également en favorisant l'augmentation de la part des activités inscrites à l'échelle communale ou à celle de l'intercommunalité. Un premier constat qui ressortait de l'analyse des mobilités actuelles était en effet que les périurbains recouraient encore trop souvent à la voiture pour des trajets de faible portée, y compris à l'intérieur de leur commune. Le second constat concernait la très faible part des activités (hors travail) réalisées à l'échelle des intercommunalités, les ménages se rendant de préférence soit dans leur commune de résidence, soit dans un pôle secondaire (Aguiléra et Thébert, 2015). II nous semblait donc que le renforcement des activités à ces deux échelles (communales et intercommunales) et leur basculement sur des modes doux constituait un enjeu important pour ces territoires.

\subsection{La proximité aux ressources du quotidien}

Nous avons centré notre réflexion sur l'influence de l'offre de commerces et services à l'échelle de la commune de résidence, eu égard aux travaux montrant une inscription croissante des activités du quotidien (hors travail) à cette échelle. À l'échelle locale, les politiques d'aménagement peuvent en effet contribuer à renforcer les tendances en cours de densification du tissu d'emplois et de commerces, et d'autonomisation des territoires périurbains (Bonnin-Oliveira et al. 2014). Ces politiques sont connues pour avoir un effet sur le choix modal des habitants (Ewing et Cervero, 2010) : des communes plus denses avec une mixité fonctionnelle plus importante et des aménagements de la voirie ad hoc ont, toutes choses égales par ailleurs, une part modale plus faible de la voiture particulière dans les déplacements quotidiens. L'augmentation des ressources locales peut par ailleurs bénéficier aux ménages ayant des difficultés de mobilité automobile, pour des raisons de santé ou économiques.

Néanmoins, dans le même temps, la préservation du cadre de vie des habitants apparaît comme un enjeu fort de politique locale (Jeanneaux, 2006) afin de préserver l'attractivité résidentielle de la commune. Or l'augmentation du nombre de commerces et services peut se heurter au désir des habitants de préserver un cadre de vie "rural ». Les communes périurbaines doivent donc choisir entre des politiques de développement local favorables à la densité d'activités, et au report modal de certains déplacements réalisés en automobile, et 
des politiques de développement local privilégiant la qualité de vie résidentielle. L'article explore cette tension en questionnant les réactions des habitants à des politiques potentielles de densification du tissu d'activités.

\subsection{L'usage des TIC}

Les relations entre les TIC et les déplacements physiques font l'objet d'un débat qui s'est trouvé récemment renouvelé avec la diffusion des téléphones mobiles et en particulier des smartphones. Si la thèse de la substitution est naïve et non étayée par les enquêtes, qui ne montrent pas de baisse du nombre des déplacements au cours des dernières années, il n'est pas exclu que dans un contexte où l'usage de l'automobile deviendrait plus difficile (pour des raisons budgétaires ou parce que des politiques restrictives seraient mises en place ou encore en cas de forte aggravation de la congestion des réseaux) les individus se saisissent des technologies pour diminuer leur usage de la voiture (Aguiléra et al., 2012).

Les domaines du travail (Metzger et Cléach, 2004 ; Aguiléra et al., 2014) et des achats sont en première ligne, d'autant plus qu'il s'agit des deux principaux motifs de la mobilité quotidienne : ils expliquent, en semaine, près de la moitié des déplacements des Français (CGDD, 2010). Il s'agit aussi des deux activités pour lesquelles la voiture est jugée la plus indispensable pour les ménages : en $2008,73 \%$ des actifs français utilisaient la voiture pour se rendre sur leur lieu de travail, et les distances domicile-travail des périurbains sont plus élevées que celles des urbains (CGDD, 2010). Le e-commerce constitue aussi une alternative potentiellement intéressante à la mobilité physique vers les centres d'achats, en particulier dans les territoires périurbains et ruraux, plus éloignés en moyenne des ressources du quotidien (Motte-Baumvol et al., 2012). Nous avons donc essayé d'évaluer si la mise en place de solutions (partielles, c'est-à-dire quelques jours par semaine ou par mois) de télétravail (à domicile, les télécentres étant peu répandus dans les territoires enquêtés) ainsi que la livraison des courses pouvaient être des pistes pour les ménages. Les enquêtes permettaient par ailleurs de déterminer si les ménages avaient ou non déjà recours à ces pratiques de travail et d'achats à distance.

\subsection{Des changements de mobilité aux changements de localisation}

Le passage à des pratiques de mobilité plus sobres recouvre trois grandes catégories de changements non exclusifs et qu'il s'agit de combiner au mieux (Schwanen et al., 2012) : l'abandon de pratiques de mobilité "carbonée " (par report modal ou par baisse de la fréquence des déplacements réalisés en automobile, par exemple), l'accentuation d'habitudes de mobilité " non carbonées » naissantes (croissance de la mobilité cycliste, par exemple), et enfin le développement de ce que T. Schwanen et al. (2012) appellent la " spontanéité » dans les mobilités "carbonées » existantes (par exemple, par une rationalisation sur le moment d'un déplacement motorisé en augmentant le taux de remplissage du véhicule). Ces trois catégories de réactions possibles, concourant toutes trois à réduire l'usage de l'automobile, ont été prises en compte dans cette recherche, qui ne considère donc pas que le report modal 
est la seule voie, mais que des pratiques plus sobres dans le périurbain peuvent passer également par un renforcement des déplacements de courte distance, une baisse de la fréquence des déplacements en automobile, et enfin un meilleur remplissage des véhicules.

Nous avons, plus précisément, envisagé différentes stratégies d'adaptation de la part des ménages: un regroupement des déplacements contraints afin de minimiser les distances parcourues, tout en conservant les mêmes activités (chaînage de déplacements, Boulahbal, 2000). Une autre possibilité est de réduire la fréquence de réalisation de certaines activités afin d'économiser simultanément sur le coût de ces activités et sur le déplacement à destination de ces activités. Les ménages peuvent avoir recours à diverses formes de covoiturage (Ballet et Clavel, 2007). Ils peuvent aussi changer de motorisation au profit des véhicules moins énergivores ou électriques, innovations qui sont soutenues par les politiques publiques en France (lois issues du Grenelle de l'environnement, Bozonnet et Halpern, 2013) même si leur modèle économique dans le périurbain reste à créer. Enfin, les ménages peuvent se tourner vers des modes de déplacements individuels moins coûteux, comme le scooter, le vélo ou la marche.

Nous avons, enfin, cherché à déterminer si un durcissement des conditions de mobilité (hausse des carburants) pouvait conduire les ménages à envisager des changements plus radicaux, soit en recherchant un emploi plus proche de leur lieu de résidence (Criqui et al., 2010 ; Korsu et Massot, 2006), soit en déménageant pour se rapprocher de leur emploi, et/ou d'une commune mieux équipée que la leur.

\section{Deux enquêtes complémentaires, sur trois communautés de communes}

Cet article s'inscrit dans une recherche intitulée « Dynamiques du peuplement, des formes urbaines et des mobilités dans les territoires de la périurbanisation " financée par le PUCA (Aguiléra et al., 2014). Le croisement de deux méthodologies, l'une quantitative, afin de faire le point sur les grandes tendances à l'œuvre en termes d'évolution des représentations liées à la mobilité quotidienne, et l'autre qualitative, en vue d'analyser plus finement les raisons mises en avant pour justifier ces évolutions, nous permet de proposer un regard riche sur les processus en cours, de mieux en saisir les possibles évolutions et enfin de discuter les politiques susceptibles d'encourager les habitants à des mobilités plus sobres.

\subsection{Les enquêtes}

Construites sur la base d'une même grille de questionnements, ces deux enquêtes, conduites entre 2013 et 2014, abordaient successivement (1) le parcours résidentiel des ménages et le choix de la commune actuelle, (2) les mobilités quotidiennes (identification des principaux territoires fréquentés pour les différents motifs et modes de transport associés) ainsi que la pratique du co-voiturage, du télétravail à domicile ${ }^{3}$ et du e-commerce alimentaire, et (3) les

\footnotetext{
3 Nous ne nous sommes intéressés dans les enquêtes qu'au télétravail à domicile parce qu'il s'agit de la forme de travail à distance la plus susceptible de diminuer les déplacements domicile-travail (avec le télétravail en télécentres, mais qui est
} 
possibilités en matière de changement de comportement de mobilités à travers d'une part les facteurs favorisant, du point de vue des ménages, une moindre utilisation de la voiture et, d'autre part, les réactions passées et envisagées à une hausse conséquente du prix des carburants.

Le recours à un questionnaire, auquel 300 ménages ont répondu (sur les 1800 auxquels un questionnaire a été distribué dans la boîte aux lettres entre avril et juin 2013), nous a permis d'assurer la représentativité statistique de nos résultats ${ }^{4}$, en particulier s'agissant premièrement des déplacements quotidiens que l'EGT (Enquête Globale de Transport) ne permet pas de décrire en raison d'un trop faible nombre de répondants dans les communes considérées, et deuxièmement des marges de manœuvre en matière de baisse de l'utilisation de la voiture et de hausse des coûts de la mobilité automobile.

Lors des entretiens, nous avons rencontré 32 personnes au profil varié tant dans l'âge, que dans la structure familiale, la position économique et la catégorie socioprofessionnelle. Ces entretiens semi-directifs d'une durée moyenne d'une heure, réalisés en face à face ${ }^{5}$, nous ont permis d'explorer plus finement les marges de manœuvre en matière de mobilité durable en discutant un certain nombre de tendances - dépendance automobile, mode de vie individualiste, entre-soi - qui « collent à la peau » des ménages périurbains. La première partie de la grille d'entretien reposait sur des questions extrêmement ouvertes sur le parcours résidentiel, la représentation du territoire au sens large et du lieu de résidence ainsi que leur évolution depuis l'installation. La dernière partie de la grille de l'entretien semi-directif consistait à reprendre une journée de semaine et un week-end types décrits par l'interviewé en début d'entretien, et à relever la nature des stratégies d'adaptation (portant sur la mobilité et/ou sur d'autres actions comme la diminution du budget affecté aux loisirs, aux achats, etc.). L'entretien permettait également de relever les dynamiques en cours concernant les changements de pratiques de mobilité en lien avec l'évolution des territoires et le niveau d'équipement de la commune et des communes alentour, ainsi que les représentations que la personne s'en fait.

Dans chacune de ces deux enquêtes, nous avons d'abord cherché à voir si les ménages étaient prêts et en capacité, dans les conditions actuelles, à réduire leur usage de la voiture, et à identifier quels facteurs pourraient favoriser ce changement. Dans le questionnaire, une question spécifique ${ }^{6}$ servait de support à cette réflexion : elle ne précisait pas de quel type de

\footnotetext{
encore extrêmement rare), contrairement aux formes de travail à distance en mobilité ou dans des tiers-lieux (Metzger et Cléach, 2004 ; Moriset, 2004).

${ }^{4}$ Nous disposons en effet d'un échantillon représentatif du type de ménage, du nombre de personnes par ménage, du type de logement, du statut socio-professionnel de la population résidente sur nos terrains d'étude avec toutefois une légère surreprésentation des 31-50 ans au détriment des 51-64 ans (écart = 5\%) et une surreprésentation des locataires (écart = $8 \%)$.

5 Les entretiens ont été retranscrits dans leur intégralité.

${ }^{6}$ La question était formulée ainsi : «Qu'est-ce qui pourrait vous inciter à utiliser moins votre véhicule?». Une liste de facteurs était proposée, les répondants pouvant cocher autant de facteurs qu’ils souhaitaient.
} 
modération il était question (rouler moins, utiliser les transports en commun...), car l'objectif était simplement de recueillir des premiers éléments sur les facteurs pouvant favoriser une ou des formes de sobriété automobile. Les entretiens nous permettaient, par ailleurs, d'apporter une connaissance plus fine de ces éléments.

Nous nous sommes ensuite attachés, dans chacune des deux enquêtes, à placer les ménages dans un scénario d'augmentation sensible du prix des carburants. Notre objectif était de déterminer si des stratégies étaient d'ores et déjà envisagées par les ménages, quelles étaient ces stratégies et si elles prenaient en compte les augmentations passées et récentes du prix des carburants, susceptibles en effet d'avoir déjà fait évoluer les comportements et/ou d'avoir préparé les ménages à des changements ${ }^{7}$. Les entretiens, qui proposaient notamment un scénario concret de hausse de prix, nous permettaient d'envisager d'autres stratégies d'adaptation proposées spontanément par les ménages, regroupées ici en deux catégories :

- Une baisse des dépenses de mobilité, pouvant prendre des formes variées: diminution des distances parcourues en voiture, report modal, chaînage ou regroupement des déplacements, achat d'un véhicule plus sobre, pratique du covoiturage. La relocalisation de l'emploi, mais également celle du lieu de résidence ont également été envisagées comme stratégies possibles permettant de diminuer les distances.

- Une diminution d'autres types de dépenses, en particulier dans le domaine des achats et les loisirs, faisant donc mécaniquement augmenter (ou permettant de stabiliser) la part du budget transport dans le budget des ménages.

Dans cet article nous avons essentiellement mobilisé les résultats obtenus dans la dernière partie de la grille de l'entretien qualitatif, dans laquelle nous avons cherché à explorer les représentations des facteurs pouvant conduire les ménages à réduire leur usage de la voiture, interrogé les ménages sur leurs réactions anticipées face à l'augmentation du carburant, puis les avons placés dans un scénario d'augmentation sensible du prix du carburant ( 5 à 6 euros le litre). L'exploitation du questionnaire a quant à lui permis de hiérarchiser ces résultats, et d'en garantir la représentativité statistique.

\subsection{Les territoires étudiés}

Les trois territoires étudiés ont en commun d'être à une distance quasi égale de Paris (une trentaine de kilomètres), d'avoir été tenus à l'écart de projets majeurs d'aménagement urbain ou de transport, et de former des EPCI (Établissement Public de Coopération Intercommunale). Ces deux dernières précautions servent à nous assurer que la dynamique

\footnotetext{
${ }^{7}$ Les deux questions posées dans le questionnaire étaient formulées ainsi : (1) Avez-vous modifié vos déplacements à cause de la hausse des carburants? (2) Que pensez-vous faire si les carburants continuent d'augmenter ? Pour chacune, une liste de facteurs était proposée, les répondants pouvant cocher autant de facteurs qu'ils souhaitaient.
} 
de périurbanisation avait gagné nos territoires indépendamment d'une volonté politique régionale ou nationale.

Le premier territoire (en vert sur la figure 1) est constitué des communes de la communauté de communes de la Brie Boisée (7877 habitants). Ces communes se situent en bordure sud de la ville nouvelle de Marne-la-Vallée (secteur IV), à proximité relative du RER A et de l'autoroute A4. Largement rurales il y a une quarantaine d'années, elles ont connu de fortes mutations en marge de la ville nouvelle dans un premier temps, et après la construction de Disneyland Paris dans un deuxième temps. Le deuxième territoire (en bleu sur la figure 1) est la communauté de communes de la Haute Vallée de Chevreuse (25330 habitants). Ce territoire est marqué par la présence historique d'une ligne de chemin de fer (la ligne de Sceaux désormais intégrée au RER B), dont le terminus actuel est à Saint-Rémy-lès-Chevreuse, et par l'absence de grands axes routiers structurants. L'habitat pavillonnaire côtoie des lieux de villégiature en pleine nature, et les mobilités parcourant ce territoire sont de ce fait hétérogènes. II s'agit d'une des zones où l'utilisation des transports collectifs est la plus faible, à distance égale de Paris ; ce fait peut trouver des facteurs explicatifs dans la composition sociale du territoire, très aisée. Entre ruralité et périurbanité, ce territoire nous paraît être à même d'enrichir la diversité des cas étudiés tout en interrogeant les potentiels d'évolution spécifiques de ce lieu où l'usage des transports collectifs semble difficile à encourager - du fait de l'accessibilité physique comme cognitive à ce mode de transport. Enfin, le troisième territoire (en orange sur la figure 1) est celui de la communauté de communes de Carnelle, Pays de France. II regroupe 10 communes et 20967 habitants. Le territoire n'est pas situé à proximité immédiate d'une ville nouvelle comme pour les deux autres espaces. Comme pour la Brie Boisée, on trouve une voie rapide : la N104 au sud de l'EPCl, qui offre un accès aisé aux bassins d'emploi de Cergy et Roissy. Une ligne de Transilien (ligne $H$ ) parcourt le territoire et conduit jusqu'à la Gare du Nord.

Intégrer Figure 1

Intégrer tableau 1

Dans ces territoires, la part de la voiture s'est renforcée par rapport au début des années 1980, au détriment non pas des TC, dont la part se maintient, mais de la marche et du vélo (figure 2).

\section{Intégrer Figure 2}

Ces évolutions modales ont procédé premièrement de changements dans la géographie des flux, avec une baisse, modeste, des trajets intra-communaux (très favorables à la marche) et une diminution plus soutenue des trajets vers Paris (très favorables aux TC). L'augmentation de l'usage de la voiture a également été particulièrement sensible sur les liaisons 
intercommunales, passant de $53 \%$ à $91 \%$.

\section{Les chemins vers la sobriété automobile}

Les enquêtes que nous avons réalisées confirment que la dépendance à l'automobile dans le périurbain francilien n'est pas tant fondée sur un éloignement aux ressources du quotidien que sur une articulation singulière entre un mode de vie et une organisation territoriale largement co-construites et pour laquelle les transports en commun sont largement inadaptés (Aguiléra et al., 2014). L'usage de la voiture permet d'optimiser l'accès (et notamment le temps d'accès) à des ressources principalement situées dans un rayon d'une quinzaine de kilomètres et que la voiture permet d'abord de découvrir, puis d'utiliser et finalement de hiérarchiser : 89\% des personnes interrogées par questionnaire estiment que la voiture leur permet de découvrir de nouveaux endroits. L'automobilité permet aussi d'instaurer et de maintenir une flexibilité des modes de vie que les habitants valorisent et sur laquelle ils ne souhaitent majoritairement pas revenir. A l'heure actuelle, l'automobile est l'outil nécessaire au « savoir faire territorial » (Thébert et al., dans ce numéro) des périurbains.

Cette relation singulière entre automobilité et territoire n'apparaît malgré tout ni tendue, ni surtout figée: seule une minorité des personnes que nous avons interrogées par questionnaire (23\%) considère qu'elle ne peut ou ne souhaite pas réduire son usage de la voiture (tableau 2), ce que les entretiens confirment. Plus que des facteurs socioéconomiques, c'est l'intensité de l'usage de la voiture qui pèse sur la propension à changer ou pas de comportement : ceux qui estiment ne pas pouvoir ou vouloir diminuer leur usage de la voiture roulent en moyenne 20 kilomètres par semaine de moins que les autres. Les entretiens confirment, par ailleurs, que les motivations au changement sont rarement écologiques, mais relèvent surtout de la pénibilité (fatigue et stress générés par la conduite) et, dans une moindre mesure, des implications financières du tout automobile.

Parmi la liste proposée aux ménages dans le questionnaire, quatre facteurs favorables à une baisse de l'automobilité ont été cochés nettement plus souvent que les autres. Ils sont également ceux qui ressortent le plus souvent des entretiens. Ces facteurs représentent donc les principales évolutions de contexte géographique, personnel ou professionnel qui sont perçues par les enquêtés comme les plus favorables à une réduction de leur usage de la voiture (tableau 2). La première est une réorganisation profonde de l'offre de transports collectifs, visant une meilleure adéquation avec les rythmes et la géographie des mobilités périurbaines, et en particulier des trajets domicile-travail. La deuxième évolution concerne une flexibilisation des liens entre travail et lieu de travail permettant à une partie des actifs de travailler à domicile un ou quelques jours dans la semaine, de façon régulière ou non. La troisième évolution est une hausse sensible du prix des carburants, dont nos travaux montrent toutefois un certain nombre de limites en l'absence d'améliorations en matière d'alternative à l'autosolisme. Enfin, le développement des ressources locales (commerces et services du quotidien), notamment à l'échelle communale, constitue un quatrième facteur favorable à 
une baisse de l'automobilité en permettant la poursuite de la dynamique actuelle d'inscription de la vie quotidienne des périurbains (hors activités professionnelles) dans leur territoire de résidence.

\section{Intégrer Tableau 2}

\subsection{Le potentiel d'une réorganisation profonde du système de transports en commun}

L'usage des transports en commun est, sans surprise, très faible dans nos trois territoires d'étude (figure 2). Il est, par ailleurs, cantonné à des destinations précises, qui sont principalement Paris et la petite couronne. Les trajets domicile-travail y tiennent une place importante en raison des difficultés de circulation sur le réseau routier pendant les heures de pointe en direction du cœur de la métropole.

Hormis à l'intérieur du périmètre communal, où la marche est plébiscitée, la voiture assure l'essentiel des trajets du quotidien, en particulier à destination des pôles périphériques d'emplois et de commerces qui maillent désormais la petite et la grande couronne, et dont font notamment partie les Villes Nouvelles (Aguiléra, 2005 ; Berroir et al., 2005). L'usage de la voiture est justifié par les habitants par l'absence d'offre alternative aux transports en commun ou, lorsque cette offre existe, par ses médiocres performances sur le trajet aller ou, plus souvent, retour : en particulier les bus ne fonctionnent en général que sur des plages horaires restreintes, notamment le soir. La crainte de manquer le dernier bus en revenant du travail est un frein important pour certains actifs. Anne, 42 ans, mariée et femme au foyer, habitante de la Brie Boisée, explique :

"On devient dépendant, après, donc, le voisin, c'est pareil. On s'est retrouvé... lui aussi, il a une deuxième voiture... mais il l'avait fait avant nous, il en avait pris une deuxième avant nous parce qu'il a craqué avec les bus, et... nous, on a tenu un petit peu plus longtemps et puis jusqu'au moment où ça ne devenait plus possible, quoi. (...) Non, mais parce qu'en fait, le souci, c'est que le bus... le dernier qui ramène au village, il part du Val d'Europe à 19 h40, quelque chose comme ça, et... ben, déjà, dans les transports, pour arriver jusqu'ici, il y a à peu près trois quarts d'heure, 1 heure, et du coup, ça veut dire qu'il faut qu'il parte, dernier carat, 17 h30, machin, et c'est... ça ne devient plus possible, quoi... enfin, avec le bus, ça lui pose des problèmes, donc, il a pris une voiture et en fait, il la laisse à la gare de Torcy. "

Le recours à la voiture permet, par ailleurs, de compenser la faible densité et la relative dispersion de l'offre territoriale de services, commerces et loisirs autour de la commune de résidence. Si le territoire périurbain n'offre pas encore la diversité des ressources présentes dans les centres urbains, ce n'est pas pour autant que ces habitants souhaitent renoncer à un mode de vie axé sur la diversité des activités et de l'offre. Pour obtenir un tel mode de vie, l'automobile est l'instrument indispensable de pratiques marquées par une certaine souplesse 
(horaires et lieux de destination) et une large flexibilité pour les déplacements non contraints.

Un élément important est que les enquêtés attachent une grande importance à ne pas rentrer dans un programme routinier. Cet attachement à la flexibilité de leur planning essentiellement dans le cadre des activités hors travail ${ }^{8}$, est constitutif de leur mode de vie et s'exprime par : la fréquentation d'une variété de lieux d'achats dans le cadre des courses alimentaires et du shopping et non une fidélisation à une enseigne ${ }^{9}$; mais également le fait de ne pas avoir un jour ou un horaire fixe dans la semaine pour réaliser ses activités. Hormis les activités de loisir pour lesquelles les répondants sont prêts à s'engager sur une pratique hebdomadaire, à heure fixe, les habitants fuient un programme routinier pour les autres activités, en particulier celles effectuées à l'extérieur de l'échelle de proximité (déplacement domicile-travail, courses alimentaires, démarches administratives). L'indicateur privilégié dans le choix du commerce repose sur le rapport coût du produit/distance. Or les entretiens révèlent que cet aspect de leur mode de vie est fortement valorisé par les ménages périurbains.

Cette revendication de flexibilité saisie à travers les entretiens qualitatifs, qu'on pourrait penser être surtout associée à un type de ménage spécifique (celui des ménages avec de jeunes enfants ayant un emploi du temps plus contraint que les autres), est en réalité partagée par les retraités et les célibataires de notre échantillon. Agathe, 48 ans, mariée et femme au foyer, habitante de la Brie Boisée, explique :

"Voilà. Soit je vais faire des courses directement à... à Carrefour, celui de Collégien, sinon j'allais parfois faire des courses chez Leader Price, mais plus maintenant parce qu'il a beaucoup augmenté ses prix. "

Eléonore, 34 ans, mariée et informaticienne habitant en Vallée de Chervreuse, confirme :

"C'est pareil en termes de fréquence. Ce n'est pas très... ce n'est pas quelque chose de... Oui, ça arrive, mais ce n'est pas... ce n'est pas quelque chose qui est réglé comme du papier à musique, genre, on sort tous les vendredis soirs. "

Les flux réalisés à l'échelle intermédiaire sont difficiles à massifier, puisqu'à la recherche de flexibilité et de pratiques non routinières, les habitants fréquentent une variété d'enseignes implantées sur des sites dispersés, à des moments différents. Ce n'est donc pas tant la voiture qui est plébiscitée par les périurbains, que le mode de vie et les rapports aux ressources du territoire que son usage sous-tend. Un certain nombre de désavantages de la dépendance automobile sont d'ailleurs bien identifiés par les habitants, toutes catégories sociales confondues. Ces désavantages relèvent principalement de la pénibilité des trajets

\footnotetext{
${ }^{8}$ Cette recherche de flexibilité, de maintien de liberté dans le planning quotidien s'observe également, bien que plus rarement, dans le cadre de la sphère du travail. Pour répondre à la demande de leur employeur, les enquêtés doivent s'adapter et acquérir une certaine souplesse dans les horaires de travail.

${ }^{9}$ Les lieux d'achats des périurbains apparaissent en général plus variés que ceux des résidents des pôles urbains. Ainsi, en semaine, les résidents des trois terrains d'étude réalisent 39\% de leurs achats en petit commerce, 18\% en supermarché, $11 \%$ en grande surface et $10 \%$ dans un centre commercial (les autres achats ayant lieu en hypermarché, supérette, grands magasins et marché). Cette diversité des lieux d'achats fréquentés se retrouvent moins chez les habitants des centres, ceuxci concentrant un peu plus exclusivement leurs achats dans les petits commerces et supermarchés (source : EGT 2010-11).
} 
automobiles et, dans une moindre mesure, des coûts engendrés, que la période de hausse des carburants antérieure aux enquêtes a contribué à rendre plus évidente. L'impact environnemental est peu pris en considération : en l'absence de véritable alternative, les périurbains ne se sentent majoritairement pas « coupables » de prendre leur voiture.

Dans un contexte où la mobilité est valorisée comme instrument d'autonomie et de découverte du territoire, sans que le mode de transport associé (la voiture) ne le soit véritablement, il n'est pas illogique que les périurbains franciliens expriment des attentes fortes vis-à-vis de services de transports alternatifs, en particulier sur le segment des trajets domicile-travail. Environ la moitié des personnes interrogées par questionnaire font le lien entre une réduction de leur usage de la voiture et des transports en commun mieux adaptés à leurs horaires, mais aussi, et surtout, à leurs trajets.

Il faut évidemment rester prudent et s'interroger sur la crédibilité de ces réponses : en effet notre questionnaire propose une liste de motifs préétablis, et parmi eux l'amélioration des transports collectifs est une évolution relativement peu engageante pour les individus. Ils ne perçoivent pas vraiment son coût financier (sa contrepartie en matière d'impôts), mais par contre entrevoient potentiellement son bénéfice : non seulement sur leur propre mobilité, mais probablement aussi probablement en matière d'image et d'attractivité de la commune, donc de valeur de leur bien immobilier (la plupart étant propriétaires de leur bien). Toutefois les entretiens viennent confirmer l'attente forte et partagée envers des solutions alternatives à la voiture, mais qui se calquent le plus possible sur les spécificités spatiales et temporelles du mode de vie actuel. Cette demande de « sur-mesure " n'est pas seulement la manifestation d'une mauvaise volonté des périurbains à tout changement de leur mode de vie : elle rend aussi compte de l'inadaptation du système actuel de transports en commun, qui explique que seulement $10 \%$ des personnes ayant répondu au questionnaire affirment s'être reportés sur les transports en commun lors du dernier épisode de hausse des carburants. Les gens soit ont modifié seulement à la marge leur mobilité, soit se sont appliqués à rouler moins, sans renoncer à la voiture ni se reporter vers d'autres modes.

Le fait d'avoir un emploi, donc des mobilités en moyenne plus longues, plus contraintes et aussi plus répétitives, constitue un facteur plutôt favorable au changement des mobilités: seulement $20 \%$ des actifs répondent qu'ils ne veulent ou ne peuvent réduire leur usage de la voiture, contre $31 \%$ des retraités et $27 \%$ des inactifs dont la mobilité est en moyenne moins contrainte et de portée plus courte. De façon intéressante, les employés et ouvriers sont plus nombreux que les cadres ( $28 \%$ contre $11 \%$ ) à répondre ne pas envisager se passer de l'automobile, ce qui peut soit témoigner d'une dépendance accrue à l'automobile dans les déplacements quotidiens, soit d'une culture différente de la mobilité ${ }^{10}$. L'enjeu de l'amélioration de l'offre de TC porte en particulier sur la desserte des pôles d'emploi par des

\footnotetext{
${ }^{10}$ Notons à ce sujet que pour les ouvriers, la distance moyenne parcourue continue à augmenter en IDF, dans un contexte de forte régression des distances à l'emploi. Les emplois de cadres sont relativement polarisés alors que les emplois ouvriers sont nettement plus dispersés.
} 
bus permettant d'en parcourir toute l'étendue. Les travaux de Barr et Prillwitz (2012) montrent que la possibilité d'offrir une expérience " satisfaisante " d'utilisation des transports en commun joue davantage sur les représentations des différents modes de transport que d'autres mesures comme l'augmentation du prix du carburant par exemple. Les transports publics ne peuvent bien sûr pas être présents avec la même qualité de service dans tous les territoires, mais ces résultats auxquels notre enquête fait écho invitent à placer l'usager au cœur des changements à effectuer dans l'offre de service.

Pour les autres motifs de la mobilité (notamment les achats et les loisirs), soit les habitants utilisent déjà les ressources communales (à pied ou en vélo), soit ils vont dans les pôles périphériques (en voiture). Dans ce deuxième cas la variabilité et la volatilité des horaires et des lieux fréquentés, couplées aux contraintes de déplacement en famille et, pour le trajet retour, au transport de charges, rendent difficile l'organisation de TC lourds. Ces constats n'excluent cependant pas la mise en place de solutions plus souples, mais les ménages de nos terrains n'y étaient pas sensibilisés au moment de l'enquête : le transport à la demande a été cité par seulement 1 répondant sur 7. La situation a probablement évolué depuis avec la médiatisation d'un certain nombre de nouveaux services à la mobilité basés notamment sur l'usage des smartphones.

Le " développement et la sécurisation des pistes cyclables à destination des communes des alentours " sont des pistes sérieuses, mentionnées par près du cinquième des habitants comme un facteur favorable à un moindre usage de la voiture et par plus de la moitié des entretiens qualitatifs en particulier par les ménages avec enfants. Pour l'instant, les pistes cyclables sont rares, et la pratique du vélo en dehors des limites communales ou des espaces naturels est jugée trop dangereuse.

L'option du co-voiturage est par contre assez peu mentionnée par les ménages enquêtés. Elle est d'ailleurs peu pratiquée, ou dans des conditions bien précises, notamment dans le cadre de l'accompagnement des enfants à des activités extra-scolaires, des pratiques associatives et sportives ou alors pour rendre service à un voisin âgé non motorisé (Thébert et al., dans ce numéro). Le co-voiturage semble nécessiter un temps d'adaptation et pâtit du fait que les ménages identifient bien ses désavantages (perte de flexibilité dans l'organisation des activités, difficulté de trouver quelqu'un qui fait le même trajet aux mêmes horaires, établissement de liens jugés contraignants avec des tiers, etc.), alors que les avantages ne leur semblent pas encore évidents sur de courtes distances (ou trop faibles). La hausse récente des prix des carburants, intervenue peu avant nos enquêtes, n'a d'ailleurs pas été spécialement favorable au co-voiturage : seulement $2 \%$ des ménages ont opté pour cette solution face à l'augmentation des prix. On peut malgré tout imaginer qu'une hausse plus forte et surtout plus pérenne pourrait changer la donne, ainsi que le développement de services de covoiturage dynamique. Michel, 66 ans, marié et retraité, résidant dans la Communauté de Communes de Carnelle-Pays de France, explique : 
(Le co-voiturage) "Est-ce que j'en aurais envie ? C'est ça, le problème, est-ce que... Ben, ce qui m'aiderait, c'est que finalement, peut-être que ça va devenir une obligation compte tenu du fait que vous allez avoir le prix de l'essence qui va monter d'une façon vertigineuse dans les années qui viennent. C'est... ça sera la seule solution.»

Ces propos sont corroborés par Bernard, 65 ans, retraité habitant dans la même Communauté de Communes :

"Nous ne sommes pas non plus réticents au co-voiturage, mais il faudrait que des choses se mettent en place pour se mettre en connexion avec d'autres intéressés pour les mêmes trajets. "

Le discours est le même chez Gabrielle, 34 ans, mariée et responsable des ressources humaines qui réside également à Carnelle - Pays de France :

"On essaierait, je pense, oui, de... de réfléchir un peu autrement et d'optimiser, oui. Ou alors, essayer de... effectivement... de s'accorder un peu plus avec les mamans des alentours pour essayer de faire un co-voiturage, quoi. "

\subsection{Flexibilisation des liens entre travail et lieu de travail via l'option du télétravail à domicile}

Les résultats de notre enquête ${ }^{11}$ convergent avec ceux de Berger et al. (2014) : ils révèlent que la mobilité résidentielle des périurbains s'effectue en priorité dans des territoires de proximité et se traduit donc moins souvent par une augmentation des distances domicile-travail. Néanmoins, les trajets domicile-travail peuvent être une cible privilégiée des mesures de réduction de la dépendance automobile dans le périurbain : d'une part ils sont en moyenne plus longs que les autres trajets, d'autre part leur pénibilité (répétitivité, perte de temps, fatigue, incertitudes sur le temps de trajet à cause de la congestion des réseaux) est décriée par les habitants. Enfin, leur régularité spatiale et temporelle rend leur organisation collective plus aisée. En plus de la piste des transports en commun, que nous venons d'évoquer, celle d'un développement des possibilités de télétravail à domicile est mise au jour par nos deux enquêtes. Plus d'un tiers (38\%) des actifs en emploi estiment ainsi que la possibilité de rester travailler certains jours chez eux leur permettrait de réduire leur usage de la voiture.

Pour l'instant, la pratique du télétravail à domicile (entendu dans notre enquête comme le fait de travailler au moins un jour par semaine à son domicile) est faible : $14 \%$ des habitants enquêtés déclarent télétravailler au moins une fois par semaine (12\% pour leur conjoint), et $7 \%$ au moins une fois par mois ( $8 \%$ pour le conjoint). Ces chiffres sont cohérents avec ceux de la dernière Enquête Nationale Transports et Déplacements de 2008 dans laquelle environ 8\% des actifs français déclarent travailler à domicile plus ou moins régulièrement. Certaines professions intellectuelles sont plus enclines à cette pratique qui ne dépend toutefois pas seulement du type de métier, mais également des règles (parfois tacites) en vigueur dans

${ }^{11}$ Pour plus de précisions sur le parcours résidentiel des ménages voir l'article de Thébert et al. dans ce même numéro. 
l'entreprise (Aguiléra et al., 2014), ou encore de " l'ambiance » du collectif de travail. Maryse, 62 ans, mariée et infirmière et qui habite la Communauté de Communes de Carnelle - Pays de France, nous explique :

"Je travaille à l'hôpital donc le télétravail ce n'est pas pour moi. (...) Mon mari est métallier, ferronnier, il est obligé d'être sur place. "- Maryse, CC CarnellePays-de-France, St Martin du Tertre, 62 ans, mariée, infirmière.

Eléonore, 34 ans, mariée et informaticienne, également habitante de la même Communauté de Communes, amène des arguments complémentaires :

"Je... Honnêtement, je ne me souviens plus pourquoi il était passé au télétravail. Je ne me souviens plus... Si, je sais. C'était parce que le contexte professionnel était tel que... il disait que ça n'avait aucune valeur ajoutée d'être... sur place, au travail... que de toute façon, il était en réunion téléphonique avec des gens qui n'étaient pas sur place... quoiqu'il advienne. Donc, ça ne changeait rien pour lui, et que tant qu'à être dans... que l'ambiance était telle aussi qu'il préférait être à la maison. "

Si l'option télétravail est peu envisagée (et d'ailleurs peu pratiquée) par les employés et les ouvriers que nous avons enquêtés, les cadres, pour des raisons probablement à la fois culturelles et de nature de l'activité professionnelle, expriment en revanche des attentes et des pratiques plus marquées : près de la moitié d'entre eux établissent en effet un lien entre le développement des possibilités de télétravail et la réduction de l'usage de la voiture.

Les attentes vis-à-vis de formes plus souples d'organisation spatiale du travail dépassent toutefois la seule sphère de la mobilité automobile : en effet les personnes qui utilisent les transports en commun pour aller travailler plébiscitent autant le télétravail comme outil de réduction de l'automobilité que ceux qui vont travailler en voiture. Cela signifie que le télétravail n'est pas envisagé seulement comme un outil de réduction de l'usage de la voiture, mais bien comme un moyen de réduire la pénibilité du trajet domicile-travail, la perte de temps associée, qu'on soit en voiture ou en TC. Le télétravail est vu comme un moyen de mieux concilier vie privée et vie professionnelle, notamment lorsque le trajet domicile-travail est long: les personnes qui voient dans le télétravail une incitation à une baisse de leur mobilité affichent en effet un temps de transport un peu plus élevé que les autres. II s'agit bien là d'attentes d'une meilleure qualité de vie grâce à une meilleure conciliation des activités et des temps privés et professionnels (Harpaz, 2000).

La mise en place de formes de travail plus souples, permettant d'éviter un ou deux allersretours par semaine, constitue donc une piste. Les salariés concernés ne sont pas nécessairement à la recherche de cadres très précis, instaurant dans leur contrat de travail un nombre de jours par semaine fixé à l'avance de télétravail, à domicile ou dans un télécentre (dont les pouvoirs publics encouragent d'ailleurs le développement dans le périurbain, notamment en Seine et Marne), mais vraisemblablement plutôt de solutions assez flexibles, leur permettant une meilleure gestion de leur mobilité une ou deux journées par semaine, et 
pas forcément de manière régulière. L'enjeu pour les entreprises nous semble donc de permettre un assouplissement de la relation entre travail et lieu de travail au moins pour certaines catégories de salariés. Les implications sur la réorganisation des tâches et sur le contrôle des salariés sont potentiellement importantes et compliquées à mettre en œuvre dans les entreprises, mais l'enjeu en matière de mobilité durable (aux plans environnemental, mais aussi social, sans parler de la baisse de congestion aux heures de pointe sur certains réseaux) semble suffisamment important pour que ce type d'évolution soit encouragé par les pouvoirs publics.

\subsection{La hausse des prix des carburants : l'automobile à tout prix ?}

Nos deux enquêtes avaient été précédées de plusieurs épisodes de hausse brève, mais néanmoins assez sensible, des prix des carburants et qui avaient été fortement médiatisées. Malgré le récent retournement de tendance, l'augmentation à terme du prix des carburants demeure un horizon réaliste (épuisement des ressources fossiles et exploitation de gisements dont l'extraction est de plus en plus coûteuse), dont l'effet pourrait cependant être contré par les nouvelles motorisations, mais aussi par l'arrivée de véhicules consommant seulement 2 litres aux 100 kilomètres. Malgré ces incertitudes, les conséquences d'une flambée des prix du carburant méritent d'être analysées, en particulier dans le périurbain.

Cette problématique était abordée selon plusieurs angles à travers nos deux enquêtes. Le questionnaire posait d'abord la question de la contribution éventuelle d'une hausse des prix sur l'usage de la voiture, puis analysait les effets réels de la hausse récente sur les comportements de mobilité. Enfin, le questionnaire et les entretiens investiguaient plus avant les effets possibles d'une poursuite de cette hausse en explorant les stratégies que les ménages comptaient mettre en œuvre, pas seulement dans le domaine de la mobilité, mais aussi en matière d'affectation de leurs ressources aux différents postes budgétaires (sous l'hypothèse que le maintien choisi ou contraint de la mobilité automobile pouvait se traduire par des coupes dans d'autres budgets).

Seul un peu plus du quart des ménages (27\%) ayant répondu au questionnaire associent hausse des carburants et baisse de l'usage de l'automobile (tableau 2). Les actifs sont sensiblement plus nombreux que les retraités et les inactifs à faire cette association. La hausse des carburants est malgré tout seulement la troisième réponse des périurbains lorsqu'ils réfléchissent aux facteurs pouvant faire diminuer leur dépendance automobile.

L'expérience passée vient il est vrai pour certains conforter l'idée que la rationalisation automobile est possible, et surtout suffit pour contrer une augmentation des prix : $39 \%$ des ménages interrogés déclarent qu'ils ont réagi à la flambée des prix en diminuant leur nombre de kilomètres hebdomadaires, soit $60 \%$ de ceux qui ont effectivement modifié leurs pratiques. On arguera que la hausse a été limitée dans le temps et n'a donc pas permis des changements plus profonds de pratiques de mobilité. C'est en partie vrai, comme le montrent les réponses concernant la poursuite de l'augmentation des prix des carburants (tableau 3 ) : les ménages 
sont en effet moins nombreux à n'envisager aucun changement de mobilité si la hausse se poursuit. Toutefois ils continuent d'envisager principalement de mieux maîtriser leur usage de l'automobile (rouler moins, quitte à regrouper des motifs et supprimer des déplacements) plutôt que d'engager des transferts modaux, qui sont de fait difficiles compte-tenu de l'organisation actuelle des transports en commun (trajets et horaires) et de la géographie des emplois, commerces et services (Desjardins et Mettetal, 2012).

Cette rationalisation de l'usage de la voiture ne sera toutefois pas indolore pour une partie des ménages : un tiers des personnes qui envisagent de rouler moins considèrent qu'il leur faudra aussi diminuer leurs dépenses pour les achats et les loisirs. Le niveau des revenus joue évidemment un rôle (Hivert et Wingert, 2010), et notre enquête le confirme : les employés et les ouvriers sont en proportion plus nombreux à anticiper de basculer certaines dépenses vers le budget transport, qui rappelons-le est désormais le deuxième poste de dépense des ménages français, derrière le logement, mais devant l'alimentation (Arthaut, 2005).

\section{Intégrer Tableau 3}

La diminution des déplacements automobiles prend la forme de différentes propositions, que les entretiens permettent de préciser et de regrouper en 3 catégories :

(1) Délaisser des motifs. Cette diminution passera par la suppression de certaines sorties, en premier lieu les sorties restaurant, shopping, visite de Paris (coûteuses en temps et en argent) et les sorties qui impliquent les déplacements les plus longs (celles des vacances). Prêts à sacrifier leurs activités de loisir, les enquêtés souhaitent, en revanche, préserver celles de leurs enfants et " conserver les activités vitales et essentielles " telles que les visites aux amis et à la famille (relations sociales). Anne, 42 ans, mariée et femme au foyer, habitante de la Brie Boisée, nous confie :

"II y aurait des sacrifices à faire, oui. Voilà, ça serait diminuer certains loisirs pour... nos activités à nous... pas celles des enfants, les nôtres... oui, sur le shopping, sur les visites de Paris, sur les restos, sur... les choses comme ça, oui. "

(2) Relocaliser des motifs. Les personnes proches de la retraite ou appartenant à un couple biactif se questionnent même sur le coût des déplacements domicile-travail et proposent deux scénarios : changer de travail pour se rapprocher du domicile lorsque cela est envisageable ou plus vraisemblablement arrêter de travailler. Concernant les personnes se déclarant prêtes à sacrifier leurs activités de loisirs, ces sacrifices pourront être compensés par la pratique d'activités plus locales et par la mobilisation de ressources de proximité. Cette adaptation se fera "le moment venu ", toutefois les répondants soulignent leur crainte de perte de liberté. Agathe, 48 ans, mariée et femme au foyer habitant également la Brie Boisée, explique :

" Ah, ben, on prendrait beaucoup plus le vélo. On ferait sans doute nos courses chez Casino... parce que...Ah oui. Ah oui... oui, c'est sûr. C'est sûr et certain, je 
réduirais mes déplacements et m'arrangerais pour avoir des activités à proximité. »

(3) Rationaliser des déplacements. Des motifs pourraient être regroupés et les déplacements chaînés. Pour certaines personnes, la rationalisation extrême passerait par l'utilisation de la voiture uniquement pour les déplacements domicile - travail avec un chaînage systématique pour les courses lors du retour au domicile. Marie-Christine, 45 ans, mariée et secrétaire résidant elle aussi dans la Brie Boisée, témoigne :

"Ah, ben, je réduirais tout. Ce qui est inutile. Ce qui n'est... pas vital, quoi. Je grouperais tout ce qui est... tout ce qui est vital, je le ferais parce que... c'est indispensable. Mais le reste j'essaierais, ou de grouper, ou de faire du covoiturage, ou de trouver des systèmes. Oui. Voilà. Pas l'activité, mais trouver le système pour continuer correctement, quoi. "

Se tourner plus massivement vers des achats sur Internet et la livraison à domicile n'est en revanche pas une option retenue par la majorité des ménages. Pour l'heure, le e-commerce se pratique principalement pour l'achat de livres, de cd, billets de transport ou de concert, mais en ce qui concerne les achats alimentaires les avis sont plus partagés (Thébert et al., dans ce numéro). Les résidents insistent sur le côté convivial des courses, la rencontre avec les commerçants et de l'autre, l'importance de voir l'apparence du produit (en particulier en ce qui concerne les produits frais) et de pouvoir le choisir en fonction de sa date de péremption. Les enquêtés soulignent le risque d'une flambée des prix de livraison à domicile si le prix de l'essence augmente. Ils rappellent également que le système de tarification de la livraison dépend du montant total des courses (plus le montant est élevé, plus celui de la livraison diminue) et que finalement les ménages au budget serré ou de petite taille, donc limités dans leur capacité d'achat, se retrouvent avec des coûts de livraison démesurés par rapport au montant des courses. Ce service est donc pour l'instant limité à une certaine catégorie de la population. Habitante de la Brie Boisée, Flora, 35 ans, mariée et qui travaille dans une banque nous explique :

"Non, je fais des achats par internet pour tout ce qui est vestimentaire, déco, mais non, jamais alimentaire. Je me déplace, j'ai besoin de voir ce que j'achète surtout pour les légumes, les produits frais. (...) Sinon je consomme des produits locaux, on a une petite agricultrice qui vend des œufs, je vais aussi souvent à la ferme à Marne et à Tournan il y a un boucher qui vend la viande de ses bêtes. "

Les propos de Vincent, 49 ans, marié et informaticien habitant la Communauté de Communes de Carnelle - Pays de France, vont dans le même sens : 
"Les courses... les courses, c'est ma femme qui les fait, au Drive à Carrefour. Donc, elle fait ça en semaine. Elle sort du travail et elle va chercher les courses et elle les ramène. "

Enfin, 16,4\% des ménages de nos terrains d'étude entrevoient par ailleurs dans l'achat d'un véhicule électrique (ou hybride) une solution pour résoudre le problème de l'augmentation du prix de l'énergie : actuellement toutefois le prix élevé et un certain nombre d'incertitudes (autonomie, bornes de recharge) constituent des freins à l'achat de véhicules électriques, mais s'ils sont levés, ces véhicules pourraient séduire une partie des ménages périurbains.

De manière générale, ces résultats montrent qu'il n'est pas envisagé par les ménages de bouleverser de façon radicale le mode de vie actuel, c'est-à-dire leur mobilité, mais également leur localisation résidentielle (Desjardins et Mettetal, 2013), non plus que celle de leur lieu d'emploi. Le déménagement n'est considéré comme une solution que dans peu de cas. Et lorsque les enquêtés parlent de déménager en réponse au scénario proposé dans les entretiens qualitatifs, ils décident de partir en province et ne souhaitent en aucun cas se rapprocher de Paris. La recherche d'un emploi plus proche n'est pas non plus une option envisagée ou crédible pour la plupart des actifs, probablement parce que le fort taux de chômage rend tout changement d'emploi délicat et aussi parce que la géographie des emplois métropolitains laisse peu de place, à localisation résidentielle inchangée, à un tel rapprochement (Korsu et Massot, 2006). La piste suggérée par Schwanen et al. (2012) concernant la rationalisation des déplacements motorisés paraît donc particulièrement adaptée dans les territoires périurbains.

\subsection{La piste des ressources locales}

L'accroissement du nombre de services et commerces dans la commune est cité en quatrième position par les ménages périurbains. Cette piste est d'autant plus crédible qu'elle s'inscrit dans une dynamique actuelle de recours croissant aux aménités locales, et notamment communales, dans des domaines variés: courses d'appoint, loisirs, mais aussi santé. Les distances entre le domicile et les lieux de réalisation favoris de ces activités sont en effet respectivement de $5 \mathrm{~km}, 1 \mathrm{~km}$ et $9 \mathrm{~km}^{12}$, cette dernière distance étant élevée essentiellement, car une petite partie des ménages citent Paris comme leur premier lieu de réalisation d'activités de loisirs.

L'augmentation des ressources locales permettrait de réduire les distances parcourues en voiture et de favoriser la marche et le vélo, qui sont les modes dominants pour les trajets intracommunaux. Les personnes qui ont le plus recours au local, c'est-à-dire les inactifs et les retraités, sont proportionnellement plus nombreux ( $27 \%$ et $31 \%)$ à établir une relation entre

\footnotetext{
12 Distance moyenne à vol d'oiseau en considérant comme nulle la distance de déplacement intra-communale, ce qui minore la valeur moyenne.
} 
les ressources locales et une forme de sobriété automobile, contre $21 \%$ des actifs qui expriment ainsi la contrainte que fait peser la localisation de leur emploi sur leurs mobilités.

Au sein des territoires enquêtés, une différence sensible apparaît entre la Brie Boisée et la communauté de communes de la Haute Vallée de Chevreuse, toutes deux dotées de façon satisfaisante en commerces de proximité : alors que les ressources de la première semblent déjà utilisées au maximum (la possibilité de faire ses courses plus près n’apparaît pas comme un levier important de rationalisation de l'usage de la voiture), les habitants de la seconde identifient à l'inverse d'importantes marges de manœuvre puisque $30 \%$ d'entre eux citent l'augmentation de l'offre commerciale locale comme levier possible. A contrario, le souséquipement en commerces et services à Carnelle - Pays de France se traduit par le fait qu'un tiers des ménages citent l'augmentation de l'offre commerciale comme levier possible de baisse d'usage de la voiture.

L'augmentation du nombre de commerces et de services à l'échelle communale semble donc constituer une piste au regard de l'objectif de sobriété automobile, en particulier pour les moins mobiles qui sont les retraités et les inactifs. Toutefois les habitants souhaitent majoritairement que cette augmentation des ressources locales ne se fasse pas au prix d'une croissance démographique, révélant ainsi le difficile équilibre à tenir pour les élus entre développement communal et maintien d'une forme de ruralité (Poulot et al., dans ce numéro) de plus en plus plébiscitée par les habitants.

\section{Conclusion}

Le recours à l'automobile dans le périurbain francilien accompagne la structuration d'un mode de vie flexible dans ses destinations et ses horaires. Il procède aussi de contraintes objectives qui concernent au premier chef la faiblesse d'offres alternatives à l'autosolisme. C'est toutefois l'automobilité et non l'automobile qui est valorisée par les périurbains, c'est-à-dire le service de mobilité rendu par la voiture et qui pour l'instant est sans équivalent dans ces territoires peu denses. Ce service n'est malgré tout pas sans contrepartie, les aspects financier et surtout écologique n'étant pas les plus importants pour l'instant pour les ménages : c'est plutôt la pénibilité (fatigue, stress, perte de temps) qui est décriée, en particulier celle associée aux trajets domicile-travail. Ces reproches constituent un contexte favorable à une baisse de l'usage de la voiture. L'ancrage croissant des activités quotidiennes des habitants dans les territoires de proximité, et notamment leur commune de résidence, constitue aussi un élément très favorable.

Cette recherche suggère trois principales pistes d'action pour réduire la dépendance automobile dans le périurbain francilien (Figure 3). 
La première concerne les déplacements domicile-travail, qui nous semblent constituer une cible privilégiée au regard de l'objectif de réduction de la dépendance automobile. Les réflexions sur l'évolution du système de transports en commun, visant à mieux l'adapter à la multipolarisation des navettes, mais aussi sur le télétravail à domicile (dans une forme très souple) semblent des options qui peuvent assez facilement rencontrer un écho parmi les ménages périurbains. La deuxième piste vise plutôt les autres motifs de la mobilité et une diminution des kilomètres parcourus en voiture : il s'agit de poursuivre le développement de l'offre locale de commerces, services et loisirs afin d'amplifier la dynamique de relocalisation des activités quotidiennes et aussi de protéger les ménages les plus modestes d'une hausse des carburants. Cette dernière évolution ne peut en l'état à elle seule faire radicalement changer les pratiques de mobilité, au moins à court et moyen termes, en l'absence $d^{\prime}$ alternatives en matière de transport ou de lieux $d^{\prime}$ achats et de services. Toutefois elle nous met sur la piste d'une troisième option, celle de l'anticipation d'une potentielle vulnérabilité des ménages à une augmentation drastique du budget transport. On voit en effet que les ménages vont soit sacrifier certaines dépenses pour continuer à utiliser leur voiture, soit rationaliser leur usage actuel en attendant une éventuelle baisse, mais sans bouleverser profondément leurs pratiques actuelles. Agir sur les différents leviers de report modal permet de préparer l'adaptation des territoires à un contexte de contraintes environnementales et économiques nouvelles: la contribution principale de cet article consiste donc en une photographie, à un instant donné, des inflexions de modes de vie qui semblent matures à l'échelle des ménages du périurbain, et de celles qui ne le semblent pas encore (transport à la demande, changement de lieu de résidence ou de lieu d'emploi, covoiturage).

\section{Bibliographie}

Aguiléra A., 2005. Growth in commuting distances in French polycentric metropolitan areas: Paris, Lyon and Marseille. Urban Studies, 42(9), 1537-1547.

Aguiléra A., Guillot C., Rallet A., 2012. Mobile ICTs and physical mobility: Review and research agenda. Transportation Research Part A: Policy and Practice, 46 (4), 664-672.

Aguiléra A., Lethiais V., Rallet A., 2014. Le télétravail: sortir de l'impasse. Métropolitiques.eu

Aguiléra A., Nessi H., Sajous P., Thébert M. (dir), 2014. Dynamiques du peuplement, des formes urbaines et de la mobilité dans les territoires de la périurbanisation. Quels enseignements au regard du développement durable ? Rapport pour le PUCA, décembre.

Aguiléra A., Thébert M., 2015. La place de la commune de résidence dans les modes de vie périurbains. Colloque "Construire les proximités dans un monde global ", Tours, 20-22 mai.

Aragau C., Berger M., Rougé L., 2011. Du périurbain aux périurbains. Diversification sociale et générationnelle dans l'ouest francilien. Pouvoirs locaux, 3(94), 58-64.

Arthaut R., 2005. Le budget transports des ménages depuis 40 ans. La domination de 
I'automobile s'est accrue. INSEE Première, $\mathrm{n}^{\circ} 1039$.

Baccaïni B., Sémécurbe F., 2009. La croissance périurbaine depuis 45 ans - Extension et densification. Insee Premiere, $n^{\circ} 1240,4$.

Ballet J.C., Clavel R., 2007. Le covoiturage en France - État des lieux et perspectives. Rapport CERTU, $86 \mathrm{p}$.

Bamberg, S., Fujii, S., Friman, M., \& Gärling, T., 2011. Behaviour theory and soft transport policy measures. Transport policy, 18(1), 228-235.

Banister D., 2008. The sustainable mobility paradigm. Transport Policy, 15, 73-80.

Barr S., Prillwitz J., 2012. Green travellers? Exploring the spatial context of sustainable mobility styles, Applied geography 32 (2), 798-809.

Beaucire F., Chalonge L., 2007. Le desserrement des emplois au sein des aires urbaines: dépendance, autonomie ou intégration pour les espaces périurbains et leurs habitants ? Annales de la Recherche Urbaine, Ministère de l'équipement. Plan urbanisme construction architecture, 97-101.

Berger M., Aragau C., Rougé L., 2014. Vers une maturité des territoires périurbains ? EchoGéo, 27, 2014, consulté le 09 février 2015.

Berroir S., Mathian H., Saint-Julien T., Sanders L., 2007. La mobilité dans la construction du polycentrisme métropolitain. In Thériault M. et Des Rosiers F.- Information géographique et dynamiques urbaines. Lavoisier, 292p.

Berroir S., Cattan N., et Saint-Julien T., 2005. La contribution des villes nouvelles au polycentrisme francilien: l'exemple de la polarisation liée à l'emploi. Espaces et sociétés, 1, 113-133.

Bonnin-Oliveira S., Berger M., Aragau C., Rougé L., Desbordes F. et al., 2014. Les "pôles secondaires" dans la réorganisation des mobilités : Maturité et durabilité des espaces périurbains? Vivre en ville hors des villes. Synthèse de programme de recherche, MELT/MEDDE/PUCA, 29-41.

Bonsall P., 2009. Do we know whether personal travel planning really works? Transport Policy, 16, 6, 306-314.

Boulahbal M-H., 2000. Les territoires de mobilité quotidienne. Définition du concept et caractérisation en fonction du contexte d'urbanisation et de caractéristiques individuelle. Thèse de doctorat ENPC sous la direction de Orfeuil J-P.

Bouleau M., Mettetal L., 2013. La mobilité dans le périurbain francilien. Perspectives pour une organisation plus durable des activités quotidiennes des ménages seine-et-marnais au sein d'un territoire peu dense. PREDIT (GO3), $110 \mathrm{p}$.

Bouleau M., Mettetal L., 2014. La mobilité dans le périurbain : désir d'ancrage et nouvelles proximités. Note rapide, $\mathrm{n}^{\circ} 646$, Institut d'Aménagement et d'Urbanisme - Ile-de-France, mars 2014.

Bozonnet J.P., Halpern C., 2013. The environmental policy assumption revisited: Explaining trends in environmental concerns in France between 1971 and 2008. French Politics, 11, 4872. 
Brutel C., Levy D, 2011. Le nouveau zonage en aires urbaines de $2010.95 \%$ de la population vit sous I'influence des villes. INSEE Première, $n^{\circ} 1374$, octobre.

Castex E., 2007. Le Transport A la Demande (TAD) en France : de l'état des lieux à l'anticipation. Modélisation des caractéristiques fonctionnelles des TAD pour développer les modes flexibles de demain. Thèse de Géographie à l'Université d'Avignon.

CGDD, 2010. La mobilité des Français. Panorama issu de l'enquête transports et déplacements 2008. Commissariat Général du Développement Durable.

Criqui P., Menanteau P., Avner P., 2010. Quels outils pour éclairer les décisions des collectivites locales dans le domaine du climat ? In Carassus J. \& Duplessis B, Economie et développement urbain durable, 19-39. Presses des Mines.

Desjardins X., Mettetal L., 2012. L'habiter périurbain face à l'enjeu énergétique. Flux, n89-90, 46-57.

Donovan S. ; Genter, J., Petrenas, B., Mumby, N., Hazledine, T., Litman, T., Hewison, G., Guidera, T., O'Reilly, L., Green, A., Leyland, G., 2008. Managing Transport Challenges When Oil Prices Rise. Technical Report for the New Zealand Transport Agency. Wellington, New Zealand.

Draetta L., 2003. Le décalage entre attitudes et comportements en matière de protection de I'environnement. In Gendron C., Vaillancourt J-G. (Dir.), Développement durable et participation publique. De la contestation écologiste aux défis de la gouvernance. Les presses Universitaires de Montréal, 70-89.

Ewing R., Cervero R., 2010. Travel and the Built Environment. A Meta Analysis. Journal of the American Planning, Volume 76, Issue 3.

Floch J.-M., Levy D., 2011. Le nouveau zonage en aires urbaines de 2010. Poursuite de la périurbanisation et croissance des grandes aires urbaines. INSEE Première, 1375, octobre.

Harpaz I., 2000. Advantages and disadvantages of telecommuting for the individual, organization and society. Work Study, 51(2), 74-80.

Hivert L., Wingert J.-L., 2010. Automobile et automobilité: quelles évolutions de comportements face aux variations du prix des carburants de 2000 à 2008 ? In Bonnafous A., Boucq E., Glachant M., Hivert L., Kaufmann V., Goyon M., Madre J.L., Ortar N., Vincent S., Wingert J.L., Pétrole, mobilité, CO2 : Les politiques publiques et l'automobilité face à la variation des prix du pétrole. Rapport Final, mai 2010, PREDIT, 7-25

Hughes J.E., Knittel C.R., Sperling D., 2008. Evidence of a Shift in the Short-Run Prince Elasticity of Gasoline Demand. Energy Journal, 29 (1), 93-114

Huyghe M., 2015. Habiter les territoires ruraux. Comprendre les dynamiques spatiales à l'œuvre, évaluer les perspectives d'évolution des pratiques de mobilité des ménages. Thèse de doctorat soutenue le 16 novembre, Université François Rabelais de Tours.

Jeanneaux P., 2006. Economie de la décision publique et conflits d'usages pour un cadre de vie dans les espaces ruraux et périurbains. Développement durable et territoires, Dossier 7 : Proximité et environnement

Kaufmann V., Tabaka K., Guidez J-M., Louvet N., 2010. Et si les Français n'avaient plus seulement une voiture dans la tête ? Edition du Certu. 
Korsu E., Massot M.-H., 2006. Rapprocher les ménages de leurs lieux de travail : les enjeux pour la régulation de l'usage de la voiture en Île-de-France. Les Cahiers Scientifiques du Transport, 50, 61-90.

Laugier R., 2012, L'étalement urbain en France. Centre de Ressources Documentaires Aménagement, Logement et Nature.

Lejoux P., Raux C., 2011. Attitudes et changements de comportement de mobilité des ménages face à l'instauration de politiques de rationnement du carburant automobile : résultats d'une enquête qualitative. Les Cahiers Scientifiques du Transport, 59, 57-82.

Le Néchet F., Aguiléra A., 2012. Forme urbaine et mobilité domicile-travail dans 13 aires urbaines françaises : une analyse multi échelle. Recherche Transports Sécurité, 28, 3-4, 259270.

Le Néchet F., 2012. Approche multiscalaire de la mobilité domicile-travail en Ile-de-France et dans la région Rhin-Ruhr. Cahiers de Géographie du Québec, 56, 158, 405-426.

Lorenzoni I., Langford I-H., 2001. Climate change now and in the future: a mixed methodological study of public perceptions in Norwich, CSERGE, ECM 01-05.

Metzger J.-L., Cléach O., 2004. Le télétravail des cadres : entre suractivité et apprentissage de nouvelles temporalités. Sociologie du Travail, 46, 4, 433-450.

Moriset B., 2004. Télétravail, travail nomade: le territoire et les territorialités face aux nouvelles flexibilités spatio-temporelles du travail et de la production. Cybergeo: European Journal of Geography, en ligne depuis le 06 février 2004, http://cybergeo.revues.org/381.

Motte-Baumvol B., Belton-Chevallier L., Schoelzel M., 2012. Les effets de la livraison à domicile sur l'accès aux produits alimentaires: le cas des grandes surfaces alimentaires et des cybermarchés de l'aire urbaine dijonnaise. Flux, 2, 88, 34-46.

Orfeuil J-P., Ripoll F., 2015. Accès et mobilités. Les nouvelles inégalités. Gollion (Suisse), Infolio, coll. " Archigraphy poche », 211.

Poulot M., et al., 2015 (article de ce numéro spécial) (sous réserve de publication)

Rocci A., 2009. Communication, information, formation: quels impacts sur les comportements de mobilité ? Entre conscience environnementale et pratiques innovantes. Rapport de recherche INRETS-DEST financée par la DGITM-MEEDDAT, p. 156.

Saujot M., 2012. La mobilité, l'autre vulnérabilité énergétique ». Policy Brief, n05/12 mars.

Schwanen T., Banister D., Anable J., 2012. Rethinking habits and their role in behaviour change: the case of low-carbon mobility, Journal of Transport Geography, 24, 522-532.

Thébert M., et al., 2015 (article de ce numéro spécial) (sous réserve de publication)

Vincent S., 2008. Les " altermobilités ": analyse sociologique d'usages de déplacements alternatifs à la voiture individuelle. Des pratiques en émergence ? Humanities and Social Sciences. Université René Descartes - Paris V. 


\section{Illustrations :}

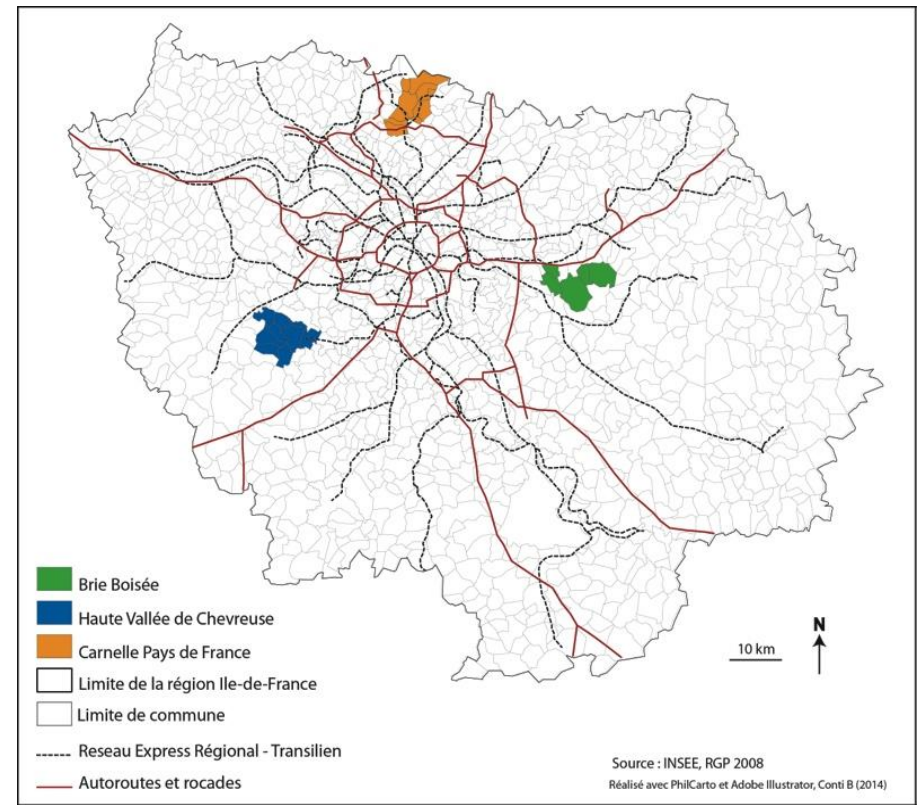

Figure 1 : Les territoires d'étude en Ile-de-France.

\begin{tabular}{|l|l|l|l|}
\hline $\begin{array}{l}\text { Nombre de } \\
\text { personnes }\end{array}$ & $\begin{array}{l}\text { Territoires } \\
\text { d'étude }\end{array}$ & Type logement & $\begin{array}{l}\text { Territoires } \\
\text { d'étude }\end{array}$ \\
\hline 1 & $22 \%$ & Maison & $81 \%$ \\
\hline 2 & $34 \%$ & Appartement & $18 \%$ \\
\hline 3 & $18 \%$ & & \\
\hline $4+$ & $27 \%$ & Statut occupation & $\begin{array}{l}\text { Territoires } \\
\text { d'étude }\end{array}$ \\
\hline & & Propriétaire & $78 \%$ \\
\hline Age & Territoires & Locataire & $22 \%$ \\
\hline$<30$ ans & d'étude & & Statut socio- \\
\hline \\
$31-50$ ans & $40 \%$ & $\begin{array}{l}\text { Territoires } \\
\text { profétude }\end{array}$ \\
\hline & & $\begin{array}{l}\text { Inactifs } \\
\text { Chômeurs }\end{array}$ & $4 \%$ \\
\hline$>65$ ans & $32 \%$ & Etudiants & $0 \%$ \\
\hline & $22 \%$ & $\begin{array}{l}\text { Retraités } \\
\text { Actifs }\end{array}$ & $\begin{array}{l}28 \% \\
66 \%\end{array}$ \\
\hline
\end{tabular}

Tableau 1 : caractéristiques socio-démographiques des territoires d'études (moyennes réalisées à partir du RGP 2010, INSEE). 


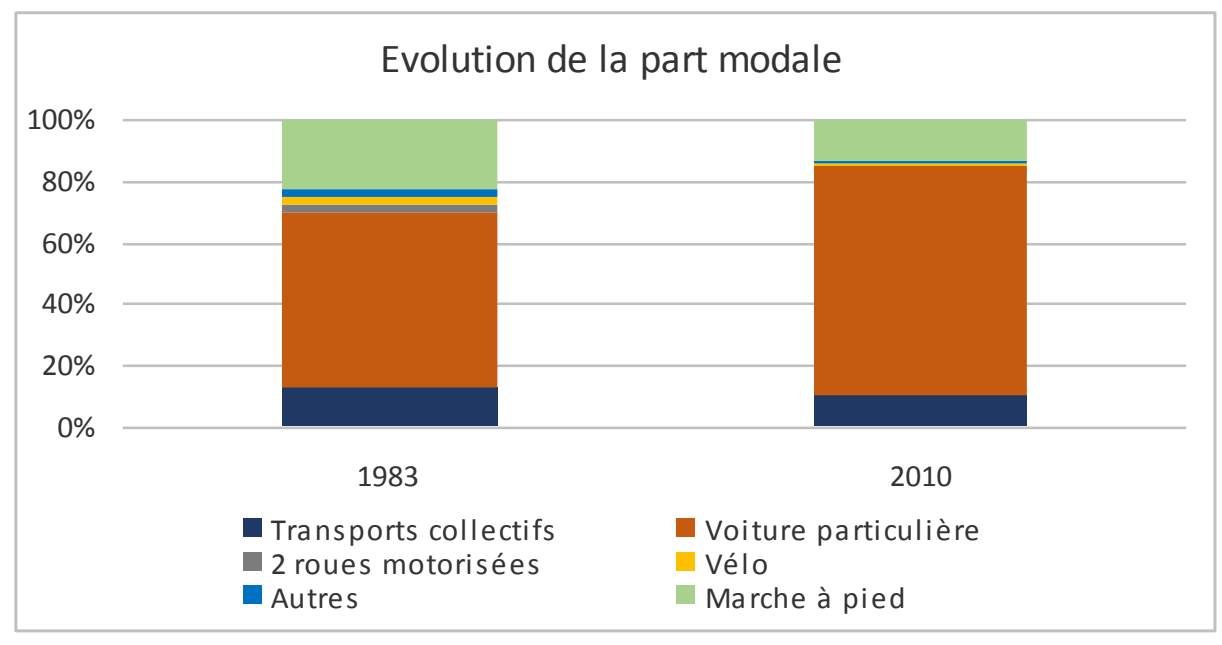

Figure 2 : Evolution de la part modale des déplacements dans les territoires d'étude. Source : Exploitation des EGT 1983 et 2010

«Q20 : Qu'est-ce qui pourrait vous inciter à utiliser moins votre véhicule ? (plusieurs réponses possibles) »

\begin{tabular}{|c|c|c|c|c|}
\hline & Ensemble & $\begin{array}{l}\text { Brie } \\
\text { boisée }\end{array}$ & $\begin{array}{l}\text { Carnelle } \\
\text { Pays de } \\
\text { France }\end{array}$ & $\begin{array}{l}\text { Vallée de } \\
\text { Chevreuse }\end{array}$ \\
\hline $\begin{array}{l}\text { Transports en commun mieux adaptés à } \\
\text { nos trajets }\end{array}$ & $53,3 \%$ & $52,4 \%$ & $53,9 \%$ & $52,9 \%$ \\
\hline $\begin{array}{l}\text { Transports en commun mieux adaptés à } \\
\text { nos horaires }\end{array}$ & $41,8 \%$ & $41,0 \%$ & $45,2 \%$ & $37,9 \%$ \\
\hline $\begin{array}{l}\text { Possibilité de travailler certains jours à } \\
\text { domicile }\end{array}$ & $26,8 \%$ & $28,6 \%$ & $21,7 \%$ & $31,0 \%$ \\
\hline Forte hausse des carburants & $26,7 \%$ & $27,6 \%$ & $25,2 \%$ & $27,6 \%$ \\
\hline $\begin{array}{l}\text { Plus de commerces et services dans la } \\
\text { commune }\end{array}$ & $23,9 \%$ & $14,3 \%$ & $31,3 \%$ & $26,4 \%$ \\
\hline $\begin{array}{l}\text { Vous ne souhaitez ou ne pouvez utiliser } \\
\text { moins votre véhicule }\end{array}$ & $23,2 \%$ & $24,8 \%$ & $23,5 \%$ & $20,7 \%$ \\
\hline $\begin{array}{l}\text { Itinéraires vélo sécurisés vers les } \\
\text { communes des environs }\end{array}$ & $16,3 \%$ & $19,0 \%$ & $13,0 \%$ & $17,2 \%$ \\
\hline $\begin{array}{l}\text { Accès à un service de transport à la } \\
\text { demande }\end{array}$ & $13,4 \%$ & $8,6 \%$ & $16,5 \%$ & $14,9 \%$ \\
\hline
\end{tabular}




\begin{tabular}{|l|l|l|l|l|}
\hline $\begin{array}{l}\text { Amélioration des cheminements pour } \\
\text { les piétons }\end{array}$ & $8,5 \%$ & $10,5 \%$ & $7,0 \%$ & $8,0 \%$ \\
\hline
\end{tabular}

Tableau 2 : Potentiel perçu de réduction de l'usage automobile. Source : enquête réalisée en 2013 auprès de 300 ménages périurbains.

\begin{tabular}{l||l} 
Principales mesures préconisées & $\begin{array}{l}\text { Individus } \\
\text { concernés }\end{array}$ \\
\hline \hline $\begin{array}{l}\text { Mise en place de formes souples de } \\
\text { télétravail }\end{array}$ & $\begin{array}{l}\text { Une partie des } \\
\text { actifs }\end{array}$ \\
\hline $\begin{array}{l}\text { Développement de formes souples de TC } \\
\text { (trajets, horaires) }\end{array}$ & Actifs \\
\hline $\begin{array}{l}\text { Développement de l'offre locale } \\
\text { (commerces, services) }\end{array}$ & Tous
\end{tabular}

Figure 3 : Synthèse des résultats. Source : Auteurs

«Q22 : Que pensez-vous faire si les carburants continuent d'augmenter ? (plusieurs réponses possibles)"

\begin{tabular}{|l|l|l|l|l|}
\hline & Ensemble & $\begin{array}{l}\text { Brie } \\
\text { boisée }\end{array}$ & $\begin{array}{l}\text { Carnelle } \\
\text { Pays de } \\
\text { France }\end{array}$ & $\begin{array}{l}\text { Vallée de } \\
\text { Chevreuse }\end{array}$ \\
\hline Rouler moins & $38,9 \%$ & $43,3 \%$ & $35,1 \%$ & $37,5 \%$ \\
\hline Ne rien changer à vos déplacements & $32,0 \%$ & $28,8 \%$ & $40,4 \%$ & $26,1 \%$ \\
\hline $\begin{array}{l}\text { Diminuer certaines dépenses pour } \\
\text { pouvoir continuer à vous déplacer }\end{array}$ & $25,6 \%$ & $24,0 \%$ & $30,7 \%$ & $20,5 \%$ \\
\hline $\begin{array}{l}\text { Prendre davantage les transports en } \\
\text { commun }\end{array}$ & $19,0 \%$ & $23,1 \%$ & $13,2 \%$ & $21,6 \%$ \\
\hline $\begin{array}{l}\text { Acheter un véhicule électrique ou } \\
\text { hybride }\end{array}$ & $16,4 \%$ & $14,4 \%$ & $14,9 \%$ & $20,5 \%$ \\
\hline Faire plus de trajets à pieds ou à vélo & $16,4 \%$ & $14,4 \%$ & $10,5 \%$ & $27,3 \%$ \\
\hline Vous faire livrer & $13,8 \%$ & $12,5 \%$ & $13,2 \%$ & $15,9 \%$ \\
\hline Faires le courses plus près & $10,8 \%$ & $6,7 \%$ & $9,6 \%$ & $18,2 \%$ \\
\hline Déménager dans une autre commune & $8,2 \%$ & $7,7 \%$ & $7,0 \%$ & $10,2 \%$ \\
\hline
\end{tabular}




\begin{tabular}{|l|l|l|l|l|}
\hline Faire du covoiturage & $7,9 \%$ & $9,6 \%$ & $7,9 \%$ & $5,7 \%$ \\
\hline Chercher un emploi plus près & $5,3 \%$ & $6,7 \%$ & $4,4 \%$ & $4,5 \%$ \\
\hline Travailler plus souvent chez vous & $4,9 \%$ & $1,9 \%$ & $5,3 \%$ & $8,0 \%$ \\
\hline Acheter une moto ou un scooter & $3,0 \%$ & $1,9 \%$ & $1,8 \%$ & $5,7 \%$ \\
\hline
\end{tabular}

Tableau 3 : Potentiel d'adaptation des ménages en cas d'augmentation des prix du carburant. Source : enquête réalisée auprès de 300 ménages périurbains.

Annexe 1: Liste des communes par terrain

\begin{tabular}{|l|l|l|}
\hline $\begin{array}{l}\text { Communauté de communes } \\
\text { de la Brie Boisée }\end{array}$ & $\begin{array}{l}\text { Communauté de communes } \\
\text { Carnelle Pays-de-France }\end{array}$ & $\begin{array}{l}\text { Communauté de communes } \\
\text { Haute Vallée de Chevreuse }\end{array}$ \\
\hline Favières & Asnières-sur-Oise & Chevreuse \\
\hline Ferrières-en-Brie & Baillet-en-France & Choisel \\
\hline Pontcarré & Belloy-en-France & Dampierre-en-Yvelines \\
\hline Villeneuve-le-Comte & Maffliers & Le Mesnil-Saint-Denis \\
\hline Villeneuve-Saint-Denis & Montsoult & Lévis-Saint-Nom \\
\hline & Noisy-sur-Oise & Milon-la-Chapelle \\
\hline & Saint-Martin-du-Tertre & Saint-Forget \\
\hline & Seugy & Saint-Lambert \\
\hline & Viarmes & Saint-Rémy-lès-Chevreuse \\
\hline & Villaines-sous-Bois & Senlisse \\
\hline
\end{tabular}

